\title{
A virtual metrology approach for maintenance compensation to improve yield in semiconductor manufacturing
}

\author{
Kuo-Yi Lin ${ }^{1}$ Chia-Yu Hsu ${ }^{2 *}$ Hui-Chun $\mathbf{Y u}^{1}$ \\ ${ }^{1}$ Department of Industrial Engineering and Engineering Management, National Tsing Hua University, \\ No. 101, Section 2 Kuang Fu Road, Hsinchu 30013, Taiwan \\ ${ }^{2}$ Department of Information Management, Yuan Ze University, No. 135, Yuan-Tang Road \\ Chungli, Taoyuan, 32003, Taiwan
}

Received 22 January 2014

Accepted 17 March 2014

\begin{abstract}
Process condition was changed by preventative maintenance that may lead to the inconsistency of process output. In practice, process engineers have difficulties to discover the inconsistency that the defect wafer may have been produced before the next measurement. This study proposes a virtual metrology (VM) approach for maintenance compensation in semiconductor manufacturing. The tool process parameter streams were collected to predict the product metrology value, and a relation model was built to adjust the equipment settings for product recipe compensation. An empirical study in maintenance compensation of chemical vapor deposition (CVD) was conducted in a Taiwan semiconductor company for validation. The thickness difference caused by maintenance was reduced from $571 \AA$ to $77 \AA$ in training data and $564 \AA$ to $210 \AA$ in testing data. The results showed practical viability of the proposed approach and an intelligent system embedded with the developed algorithm has been implemented.
\end{abstract}

Keywords: virtual metrology, manufacturing intelligence, maintenance, yield enhancement, chemical vapor deposition (CVD), semiconductor manufacturing enhancement

\section{Introduction}

Efficient management of maintenance and control for semiconductor manufacturing equipment are essential to improve productivity and yield. Maintenance action is identified by process engineer based on different policies such as Run-to-Failure (R2F) maintenance, scheduled maintenance, Condition-Based Maintenance $(\mathrm{CBM})$ and Predictive Maintenance $(\mathrm{PdM})^{1}$. Tool maintenance is conducted via monitoring of the machine or process performance with different level of complexity and efficiency. Process engineer conducts maintenance actions to improve uptime and availability and to reduce operational cost and scrap ${ }^{2}$. However, the process condition will change after the maintenance action. The change of process condition will lead to the inconsistent process output. After the further batch process of wafer manufacturing, the inconsistent process output may become a quality issue. As the device dimension shrinks further, tighter process control is needed ${ }^{3}$. The inconsistent process output may not be discovered in time and defect wafer may have been produced before the next measurement. This will result in a wafer yield loss.

To deal with this problem, virtual metrology (VM) is proposed to predict the post-process metrology variables by using the in-situ measurements and the wafer state information. As a currently practiced, VM

*Corresponding Author: cyhsu@saturn.yzu.edu.tw. 
can solve the time waste problem for waiting the metrology in the process control, but not deal with the time consuming problem for optimal equipment settings. Some existing prior literatures have been related to runto-run (R2R $)^{4,5}$, wafer-to-wafer (W2W) control $^{6}$, advanced process control framework ${ }^{7}$ and VM systems to predict faults ${ }^{8}$. However, little research has been done on maintenance compensation for yield enhancement. The temporal patterns of process variables are important signals of manufacturing behavior'. It is essential to diagnose tool effectiveness and reduce the yield loss.

This study aims to propose a VM approach for equipment maintenance compensation in semiconductor manufacturing that uses the real time tool process parameter streams to predict the product metrology value, and building the relation model to compensate values of the product recipe to adjust the equipment settings for yield enhancement. The proposed approach includes three parts. First, data preprocessing is used to signify steps within a process run and calculate summarized indicators. In this study, we generate five statistics for each tool parameters. They are standard deviation, average, range, max positive difference, and max negative difference. Second, one product metrology value is applied as the analysis target to build the prediction model for the preprocessing data and the target. Finally, a compensate model is constructed for modification of the recipe for a given process step based on the results measured on the same product at a previous process step. An empirical study was conducted in a fab for chemical vapor deposition (CVD) maintenance compensation for validation. The results showed practical viability of the proposed approach.

The remainder of this study is organized as follows. Section 2 introduces the fundamental of VM. Section 3 describes the proposed VM approach for maintenance compensation in semiconductor manufacturing. Section 4 presents an empirical study and the results for validation. Section 5 concludes this study with discussions of contributions and future research directions.

\section{Literature Review}

Real-time equipment parameter data such as temperature, pressure, gas flow, and power, were recorded as wafers pass through the process for equipment monitoring by advanced information technology and sensors in advanced $300 \mathrm{~mm}$ fabrication ${ }^{9}$. These collected equipment sensor data are related to the equipment condition and in-situ metrology such as thickness and critical dimension. Although metrology data provide useful information for $\mathrm{R} 2 \mathrm{R}$ control, however, these data are sampling due to the limited metrology capacity. Moreover, metrology data are also delay for feedback control because of wafer shipment, and measurement ${ }^{5}$. In order to measure in-situ process output for ensuring the quality, VM is a prediction of metrology variables based on the state of the equipment ${ }^{4-6}$. First, $\mathrm{VM}$ is used to predict the process quality without actually inspection ${ }^{10-12}$ in order to enhance the whole product inspection as possible. Literatures have shown the potential of VM to cope with metrology delay to wafer-to-wafer (W2W) control $^{7}$ 13. Moreover, a factory-wide control method was proposed for semiconductor manufacturing. ${ }^{5}$ In particular, VM module provides predicted metrology value based on equipment sensor data and previous metrology for run-to run (R2R) control and process control. In order to solve metrology delays, consistent process drifts, and sudden shifts in process drifts, a recursive partial least squares method for VM module was used to update the linear regression model based on the actual metrology data for semiconductor W2W control $^{6}$. The global and local VM models for plasma etch rates prediction were proposed ${ }^{14}$. In particular, windowed Gaussian process regression models has lower error of etch rate prediction than partial least square, artificial neural networks. To enhance prediction accuracy, a dynamic-moving-window scheme for VM model refreshing ${ }^{15}$. Additionally, a VM based baseline predictive maintenance scheme that possesses the capabilities of fault defection and classification and $\mathrm{PdM}^{16}$. The feasibility of real-time VM was investigated by using plasma impedance monitor data for real-time control of plasma electron density and plasma etching rate $^{17}$.

Although VM approaches have been used for process control in semiconductor manufacturing, however, little research has been done on recipe adjustment without prior measurement after equipment maintenance. In this study, VM was used to estimate the process outputs of wafers for recipe adjustment after equipment preventive maintenance. Based on the prediction of metrology value, process engineer can compensate the process outputs in advance via adjust process parameters to reduce yield loss. 


\section{Virtual Metrology Model for Maintenance Compensation}

The notations and terminologies used in this study are listed as follows:

$\varepsilon_{k} \quad$ multivariance white noise sequence, mean $=0$ and variance $=\sigma^{2}$

$\gamma \quad$ number of process output

$\xi \quad$ number of process variables

$\delta \quad$ vector of average drift rates

$\hat{\delta} \quad$ estimate of vector of average drift rates

$\sigma \quad$ standard deviation

$A \quad$ system gain matrix

$\hat{A} \quad$ estimate of system gain matrix

C coefficient matrix for the process variables

$\hat{C}$ estimate of coefficient matrix for the process variables

$i \quad$ index of wafer number after maintenance

$j \quad$ index of statistics

$k \quad$ index of process run

$n$ sample size after maintenance

Tgt target values of the process outputs

$u \quad$ process inputs or recipe settings

$u_{k} \quad$ process inputs vector that is the recipe at the start of run $k$

$\Delta u_{k} \quad$ compensate value of recipes at run $k$

$v_{k} \quad$ process variables vector at run $k$

$y \quad$ process outputs, metrology data $\hat{y} \quad$ VM conjecture value of $y$

$\mathbf{y}_{k} \quad$ process output vector obtained at the end of run $k$

The proposed VM approach for semiconductor manufacturing predictive maintenance containing three elements: maintenance and data preprocessing, virtual metrology, and process compensation, is illustrated in Fig. 1.

\subsection{Maintenance and data preprocessing}

Process engineer conducts maintenance by evaluating the condition of equipment. In the process, a large amount of data will be automatically or semiautomatically recorded in the database. Data includes process parameter which acquired from production equipment and wafer metrology after the manufacturing process. However, the collected data often include noisy, missing and inconsistent data. Thus, data preparation can improve the quality of the data to enhance the analytical results.

Data preparation includes data cleaning, data integration, data transformation and data reduction. In particular, various types of parameter cause the output variation. To identify the parameter change relates to process output variation, statistics include standard deviation, average, range, max positive difference and max negative difference were considered in the prediction model, which showed as Fig. 2.

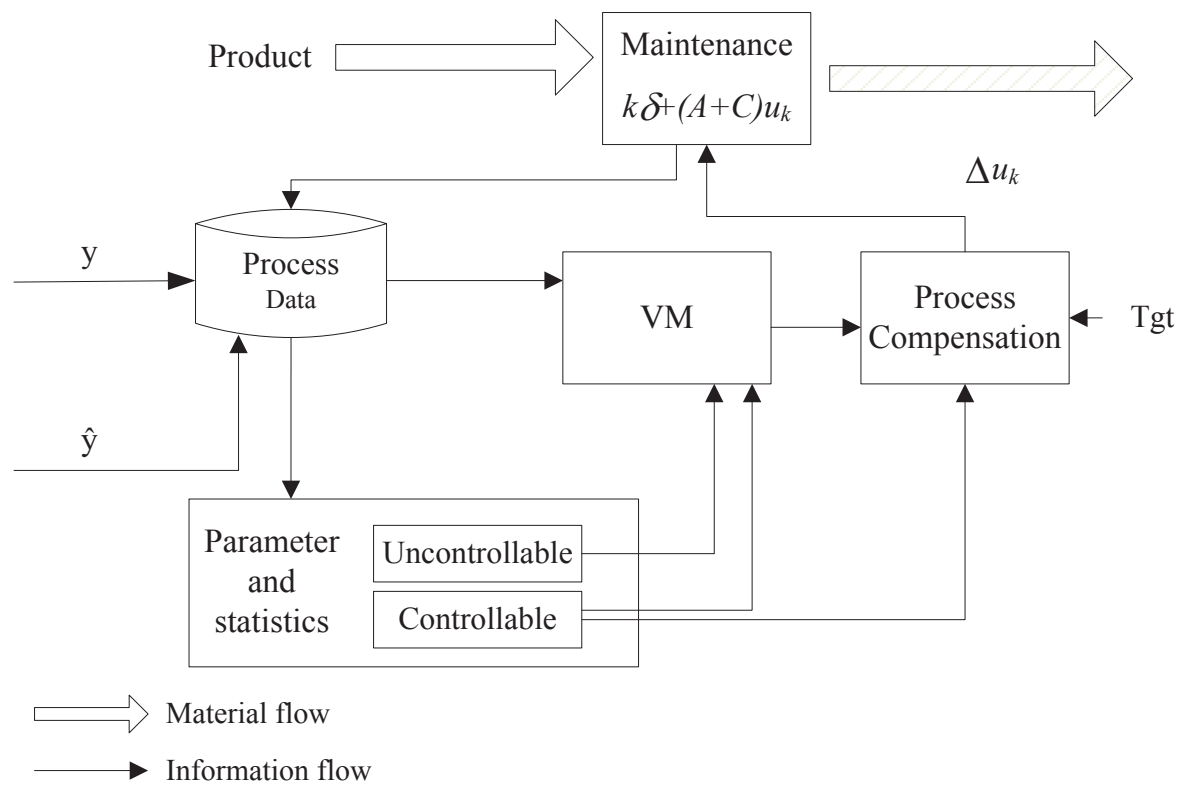

Fig. 1. VM approach for maintenance compensation in semiconductor manufacturing 

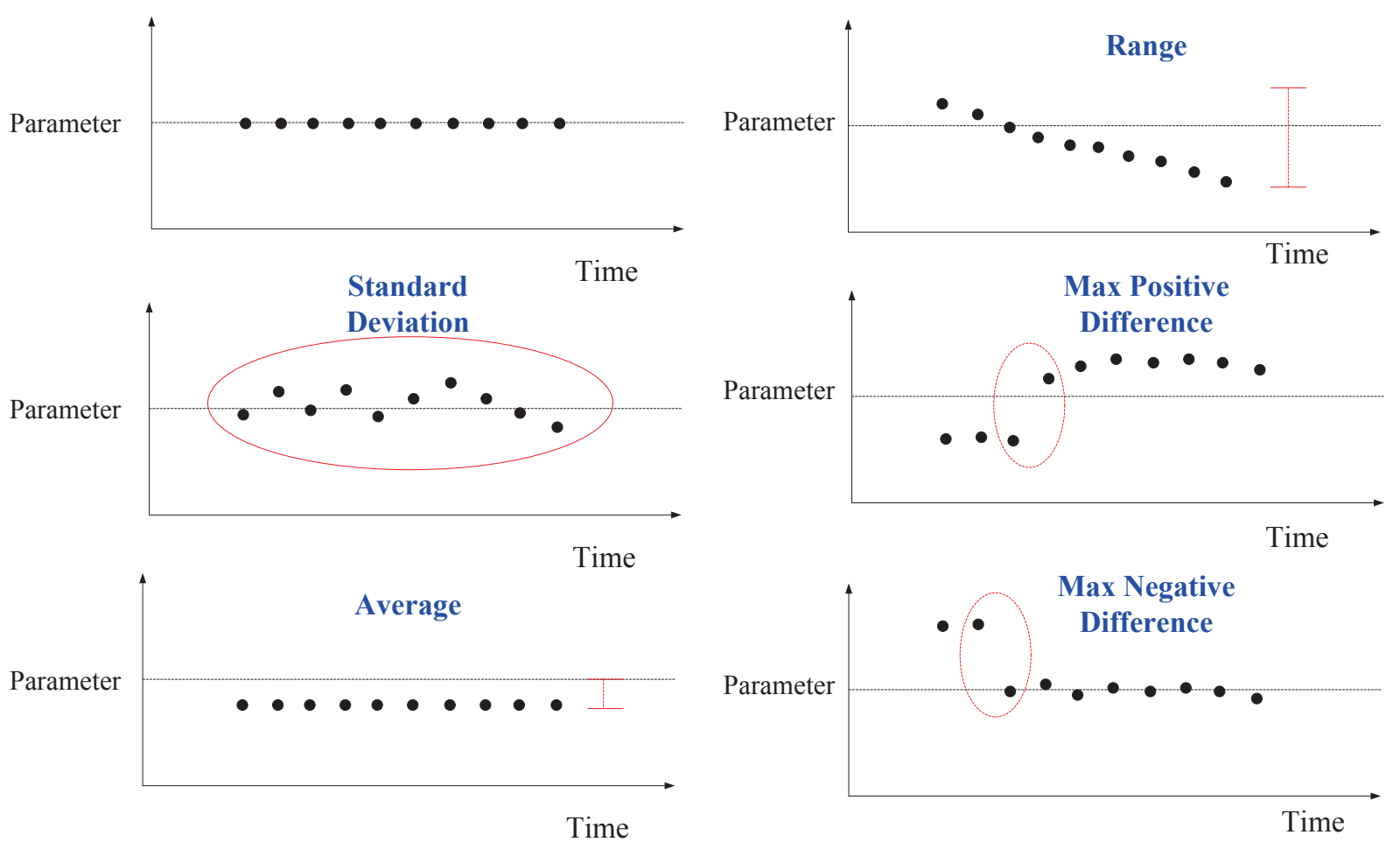

Fig. 2. Statistics for diagnosis of the parameter change

Before model construction, the analysis dataset can be partition into $\mathrm{k} \%$ training dataset and $1-\mathrm{k} \%$ testing dataset. Training data is used to derive the rules and testing data is used to estimate the validity of prediction model.

\subsection{Virtual Metrology}

The VM approach is devised for predicting the process output without actual measurement. Parameters and statistics can be divided to controllable and incontrollable. In this study, all of the combinations of parameters and statistics were being considered in the general linear multi-input-multi-output (MIMO) model to derive the relationship of process input and output.

The general linear $(\gamma \times \xi)$ MIMO approach of this paper based on ${ }^{5}$ as shown in Eq. (1).

$$
y_{k}=u_{k j} A+v_{k} C+k \delta+\varepsilon_{k}
$$

where $y_{k} \in R^{1 \times \gamma}$ is the process output vector obtained at the end of run $k, u_{k} \in R^{1 \times \xi}$ is the process inputs vector that is the recipe at the start of run $k, j$ is the index of statistics, $v_{k} \in R^{1 \times \frac{\xi}{\xi}}$ is the process variable vector at run $k$, $A$ is the system gain matrix, $C$ is the coefficient matrix for the variables, $\delta \in R^{1 \times \xi}$ is a vector of average drift rates, and $\varepsilon_{k}$ is a multvariance white noise sequence.

The prediction model was shown as Eq. (2).

$$
\hat{y}_{k}=u_{k j} \hat{A}+v_{k} \hat{C}+k \hat{\delta}
$$

where $\hat{y}_{k}$ is the estimate process output vector obtained at the end of run $k, \hat{A}$ is the estimate system gain matrix, $\hat{C}$ is the estimate coefficient matrix for the variables in the process model, and $\hat{\delta}$ is the estimate vector of average drift rates.

The accuracy calculated from the testing data is quantified by the mean absolute errors (MAE). Its formula is represented as Eq. (3):

$$
M A E=\frac{1}{n} \sum_{i=1}^{n}\left|\hat{y}_{i}-y_{i}\right|
$$

where $\hat{y}$ is the VM conjecture value of $y, y$ is the target value, $n$ is the sample size after maintenance, and $i$ is the index of wafer number after maintenance. The closer the MAE value is to zero, the better the accuracy of the model would be. The prediction results of VM should be validated by testing data and evaluated by domain experts before the process control actions. 


\subsection{Process Compensation}

The process control stage is proposed to compensate the process outputs via change process recipes, the compensate model is shown as Eq. 4. For the process control of controllable parameter and controllable statistics were considered in process compensation.

$$
\Delta u_{k j} \hat{A}=T g t-u_{k} \hat{A}-v_{k} \hat{C}-k \hat{\delta}
$$

where $\Delta u_{k}$ is the compensate value of recipes at run $k$ and $T g t$ is the target value of process outputs.

\section{Empirical Study}

To validate the proposed approach, an empirical study was conducted for a semiconductor company CVD process in a leading semiconductor company in Taiwan. CVD is a process used to develop thin film in wafer via deposit chemical via shower head. In this study, CVD chamber includes six groups of shower heads and rotating pedestal, which is shown as Fig. 3. Each wafer processes six deposition steps in the CVD process. In the process, process parameter includes chemical leak rate, power, temperature, and pressure collected by various sensors and recorded.

To avoid deformations on the wafer, it is important that parameters are performed homogeneous during the process. In this study, the engineer conducts maintenance actions to avoid the yield lose of process drift. As the prior study, the best scheduled maintenance intervention is every 45 wafers. However, maintenance induces the chamber conditions change that the deposition rate falls rapidly. There are thickness differences of wafers before and after maintenance. Fig. 4 includes one maintenance cycle data. The maintenance cycle data is the metrology data of 13 wafers which includes the thickness of wafer before maintenance (wafer 1 and 2) and the thickness of wafer after maintenance (wafer 4 14). As Fig. 4, there is a thickness difference between wafer 2 and wafer 4 . The thickness difference is $697 \AA$ that out of the given specification $(350 \AA)$ of the same lot. It is necessary to compensate the thickness difference to avoid the yield loss in the further Chemical Mechanical Polishing (CMP) process. Without losing generality, the data have been systematically transformed for proprietary information protection of the case company.

\subsection{Maintenance and data preprocessing}

Total 47 wafers with metrology data and 9 process variables process data were collected during CVD process, which include 4 maintenance cycles, each cycle include wafer thickness and 9 parameters related to chemical leak rate, Low Frequency (LF) forward power, LF load, LF reflect, High Frequency (HF) forward power, HF load, HF reflect, pressure, and temperature. In particular, five statistics for each tool parameters were considered. They are standard deviation, average, range, max positive difference, and max negative difference. Therefore, 45 statistics of parameter were be generated for construct the VM prediction model. In order to validate the prediction model, the data were split into training data (3 cycles) and testing data (1 cycle).
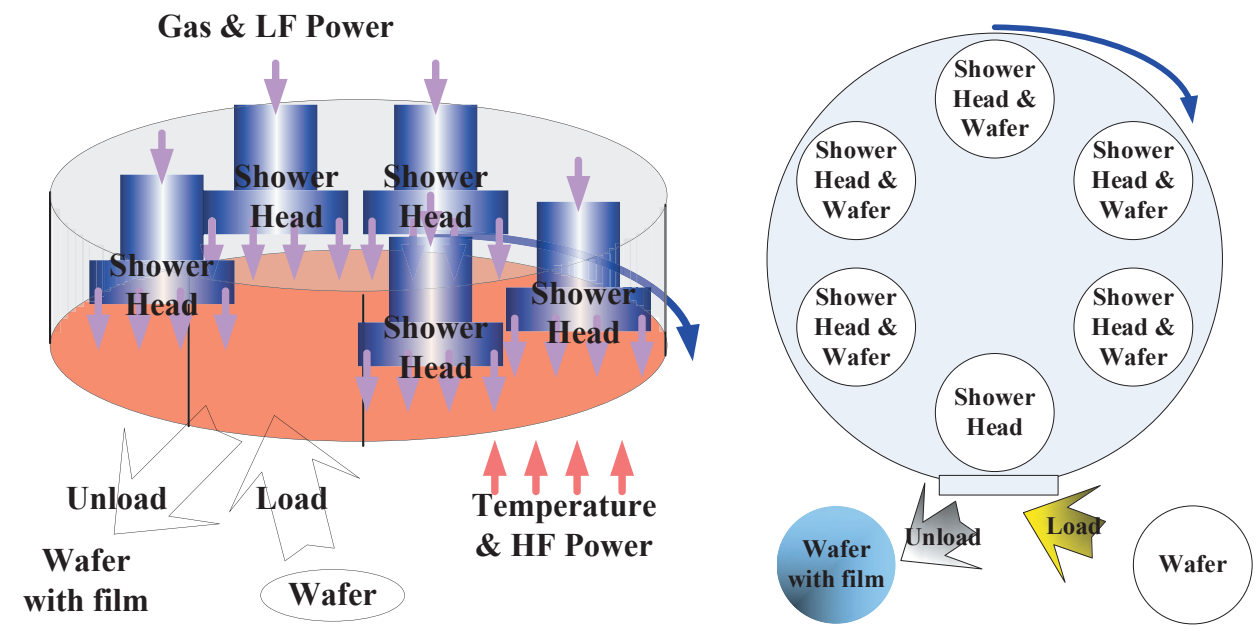

Fig. 3. CVD Chamber: scheme and horizontal section 


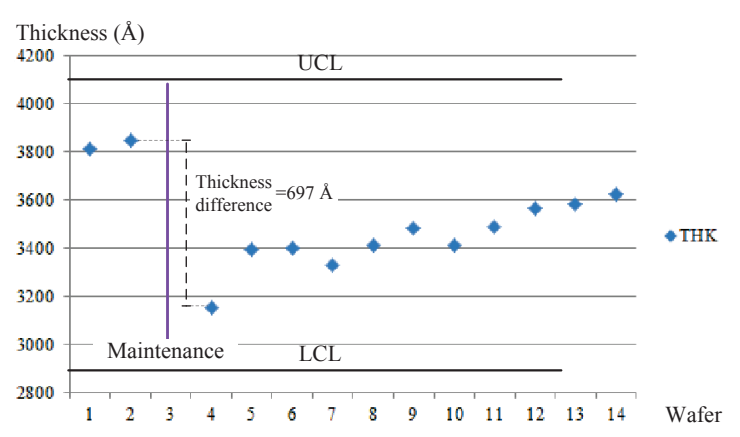

Fig. 4. Thickness differences of wafers before and after maintenance

\subsection{Virtual Metrology}

According to the general linear $(1 \times 9)$ MIMO approach the prediction model is shown as Eq. 5 .

$$
\begin{aligned}
& \hat{y}_{k}=u_{k j} \hat{A}+v_{k} \hat{C}+k \hat{\delta} \\
& =u_{\text {chemical leak rate Average }} \times 10213+460+k \hat{\delta}
\end{aligned}
$$

The prediction results of training data (maintenance cycle 1 3) and testing data (maintenance cycle 4) are shown as in Figures 5 to 8 . The MAE of maintenance Cycle 1 is shown as Eq. 6. The MAE of various maintenance cycles listed in Table 1.

$$
\begin{aligned}
& M A E_{\text {maintenance Cycle } 1}=\frac{1}{n} \sum_{i=1}^{n}\left|\hat{y}_{i}-y_{i}\right| \\
& =\frac{1}{9}(|-36.27|+|-211.71|+|115.21|+|8.89| \\
& +|110.98|+|183.01|+|20.45|+|-22.73|+|-55.42|) \\
& =84.97
\end{aligned}
$$

Table 1. MAE in various maintenance cycles

\begin{tabular}{rrrcc}
\hline & Cycle 1 & Cycle 2 & Cycle 3 & Cycle 4 \\
\hline MAE & 84.97 & 94.5 & 96.5 & 98.1 \\
\hline
\end{tabular}

The MAE of training data and testing data are consistent that shows the validity of the proposed prediction model. As the results, the average error of maintenance cycles is low, which means the accuracy of prediction model is high. Process engineers can identify the inconsistent process output without interrupt the process.

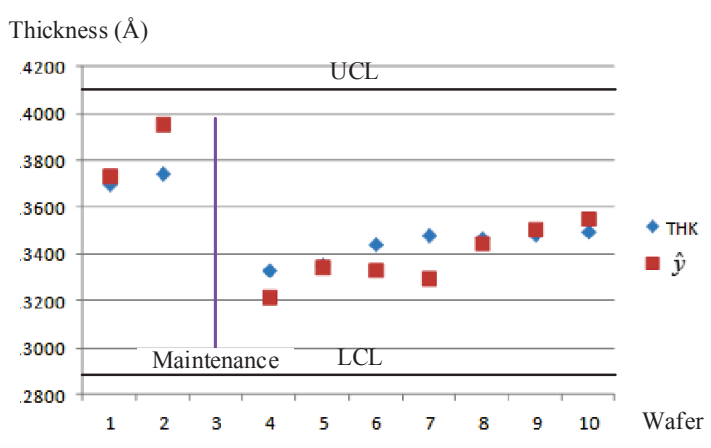

Fig. 5. Maintenance Cycle 1 results of prediction model

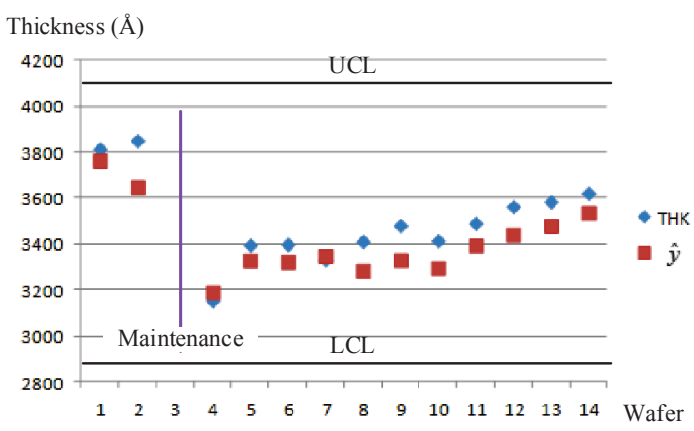

Fig. 6. Maintenance Cycle 2 results of prediction model

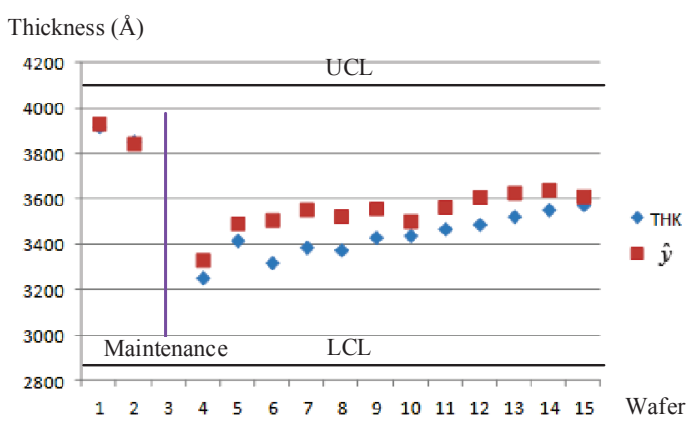

Fig. 7. Maintenance Cycle 3 results of prediction model

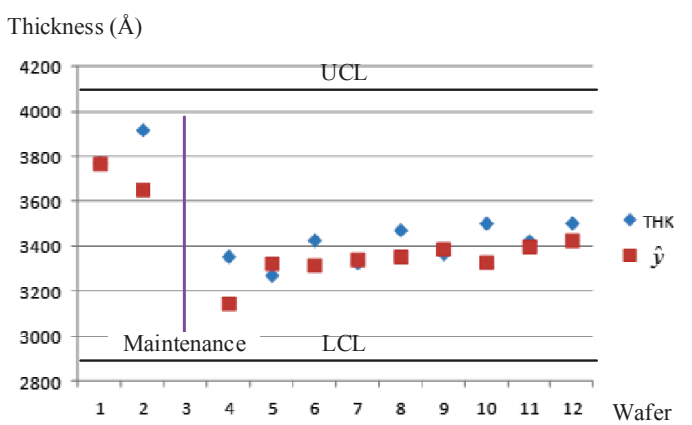

Fig. 8. Maintenance Cycle 4 results of prediction model 


\subsection{Process Compensations}

According to the discussion with domain engineers, the results in maintenance cycles 1 to 4 were shown as Figures 9 to 12. Decision support was provided before the each maintenance. Process engineers can change the recipe base on the decision support table to compensate the thickness variation to reduce the thickness difference via the intelligent system embedded with the developed algorithm. After the process compensation, the thickness of maintenance cycles were performed from THK to improved THK. The thickness difference of improved THK is much smaller than the thickness difference of THK. Table 2 showed the thickness difference caused by maintenance was reduced from $571 \AA$ to $77 \AA$ in training data and $564 \AA$ to $210 \AA$ to testing data.

Table 2. Improve rate of proposed approach

\begin{tabular}{|c|c|c|c|c|}
\hline & \multicolumn{3}{|c|}{ Training } & \multirow{2}{*}{ Testing } \\
\cline { 2 - 4 } & $\mathrm{C} 1$ & $\mathrm{C} 2$ & $\mathrm{C} 3$ & \\
\hline Actual Difference $(\AA)$ & 413 & 602 & 697 & 564 \\
\hline Control result $(\AA)$ & -115 & 79 & 36 & -210 \\
\hline Improve Rate & $72 \%$ & $87 \%$ & $95 \%$ & $62 \%$ \\
\hline
\end{tabular}

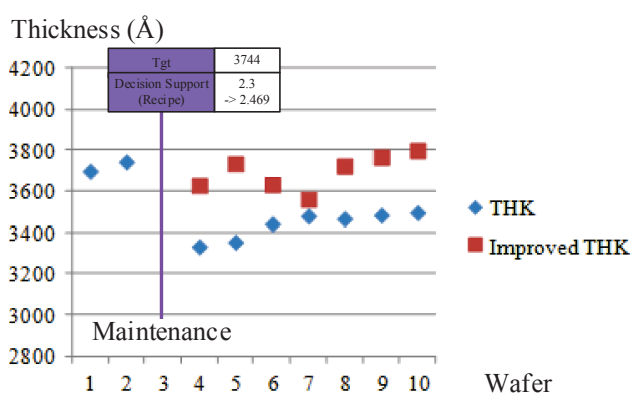

Fig. 9. Decision support table of Maintenance Cycle 1 and Improved results

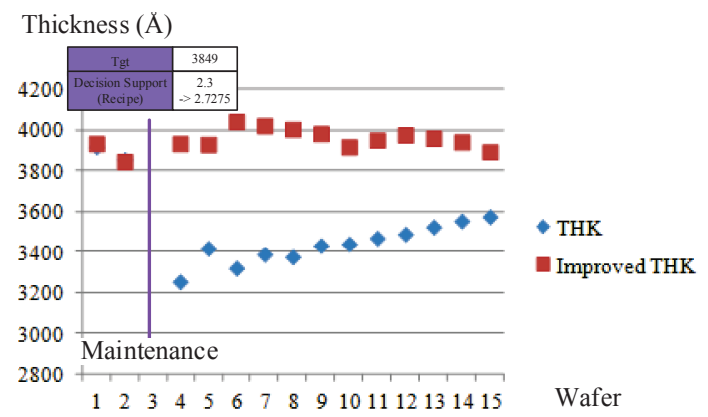

Fig. 10. Decision support table of Maintenance Cycle 2 and Improved results

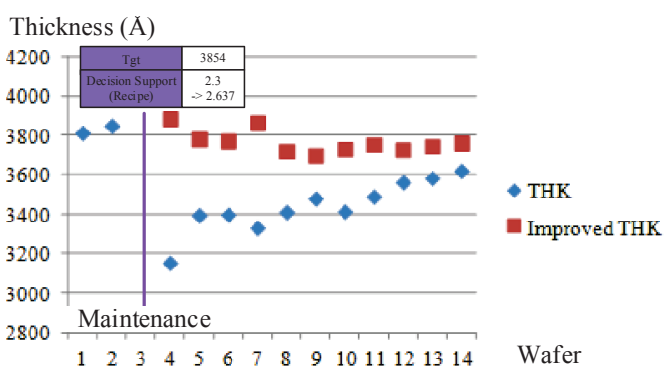

Fig. 11. Decision support table of Maintenance Cycle 3 and Improved results

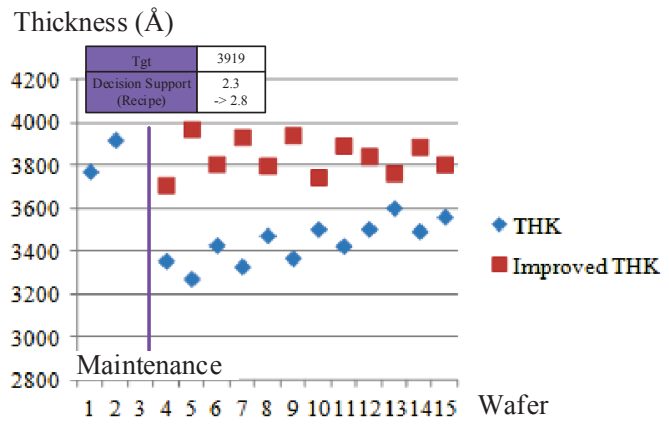

Fig. 12. Decision support table of Maintenance Cycle 4 and Improved results

\section{Conclusions}

Effective maintenance is critical to prevent yield losses from particle contamination and process drift, while maintaining tool productivity. However, the wafer thickness after CVD processing falls rapidly after maintenance that causes extra cost and time. This study proposed a VM approach to reduce thickness variance by adjusting recipes to compensate the thickness difference. The analysis results have shown practical viability of the proposed approach for yield enhancement and reduce the loss.

Further studies can be done to extend the proposed approach to the tools in other process steps and thus derive know-how for implementing the proposed approach for virtual metrology and effective maintenance for advanced equipment control. Future research can also be done to employ data mining to analyze big data of related equipment factors that can be collected in advanced wafer fabs to extract useful manufacturing intelligence for maintenance and yield enhancement $^{18-20}$. 


\section{Acknowledgements}

This research is supported by National Science Council, Taiwan (NSC102-2221-E-155-093), Hsinchu Science Park (102A26), and Macronix (100A0258JC).

\section{References}

1 G.A. Susto, A. Beghi and C. DeLuca, A predictive maintenance system for epitaxy processes based on filtering and prediction techniques, IEEE Trans. Semicond. Manuf. 25(4) (2012) 638-649.

2 A. Chen and J. Blue, Recipe-independent indicator for tool health diagnosis and predictive maintenance, IEEE Trans. Semicond. Manuf. 22(4) (2009) 522-535.

3 C.-A. Kao, F.-T. Cheng, W.-M. Wu, F.-W. Kong and H.H. Huang, Run-to-run control utilizing virtual metrology with reliance index, IEEE Trans. Semicond. Manuf. 26(1) (2013) 256-261.

4 J. Moyne, E. del Castillo and A. M. Hurwitz, Run-to-Run Control in Semiconductor Manufacturing (Boca Raton, FL: CRC, 2001).

5 A.A. Khan, J.R. Moyne and D. M. Tilbury, An approach for factory-wide control utilizing virtual metrology, IEEE Trans. Semicond. Manuf. 20(4) (2007) 364-375.

6 A. A. Khan, J. R. Moyne and D. M. Tilbury, Virtual metrology and feedback control for semiconductor manufacturing process using recursive partial least squares, J. Process Control 18(10) (2008) 961-974.

7 S.K. Fan and Y.J. Chang, An integrated advanced process control framework using run-to-run control, virtual metrology and fault detection, J. Process Control, 23(7) (2013) 933-942.

8 F.-T. Cheng, H.-C. Huang and C. A. Kao, Developing an automatic virtual metrology system, IEEE Trans. Autom. Sci. Eng. 9 (1) (2012) 181-188.

9 C.-F. Chien, C.-Y. Hsu and P.-N. Chen, Semiconductor fault detection and classification for yield enhancement and manufacturing intelligence, Flex. Serv. Manuf. J. 25(3) (2013) 367-388.

10 F.-T. Cheng, H.-C. Huang and C.-A. Kao, Dual-Phase Virtual Metrology Scheme, IEEE Trans. Semicond. Manuf. 20(4) (2007) 566-571.

11 F.-T. Cheng, Y.-T. Chen, Y.-C. Su and D.-L. Zeng, Evaluating reliance level of virtual metrology system, IEEE Trans. Semicond. Manuf. 21(1) (2008) 92-103.

12 Y.-C. Su, T.-H. Lin, F.-T. Cheng and W.-M. Wu, Accuracy and real-time consideration for implementing various virtual metrology algorithms, IEEE Trans. Semicond. Manuf. 21(3) (2008) 426-434.

13 A. Weber, Virtual metrology and your technology watch list: Ten things you should know about this emerging technology, Future Fab Int., 22 (2007) 52-54.

14 S.A. Lynn, J. Ringwood and N. MacGearailt, Global and local virtual metrology models for a plasma etch process, IEEE Trans. Semicond. Manuf. 25(1) (2012) 94-103.

15 W.M. Wu, F.T. Cheng and F. W. Kong, Dynamicmoving-window scheme for virtual-metrology model refreshing, IEEE Trans. Semicond. Manuf. 25(2) (2012) 238-246.
16 Y.S. Hsieh, F.-T. Cheng, H.-C. Huang, C.R. Wang, S.C. Wang and H.C. Yang, VM-based baseline predictive maintenance scheme, IEEE Trans. Semicond. Manuf. 26(1) (2013) 132-144.

17 S.A. Lynn, N. MacGearailt and J. V. Ringwood, Realtime virtual metrology and control for plasma etch, $J$. Process Control, 22(4) (2012) 666-676.

18 C.-F. Chien, W.-C. Wang and J. Cheng, Data mining for yield enhancement in semiconductor manufacturing and an empirical study, Expert Syst. Appl. 33(1) (2007) 192198.

19 C.-F. Chien, S.-C. Hsu and Y.-J. Chen, A system for online detection and classification of wafer bin map defect patterns for manufacturing intelligence, Int. J. Prod. Res. 51(8) (2013) 2324-2338.

20 H.-C. Yu, K.-Y. Lin and C.-F. Chien, Hierarchical indices to detect equipment condition changes with high dimensional data for semiconductor manufacturing, J. Intell. Manuf. (2013) 1-11 doi 10.1007/s10845-0130785-3. 